\title{
Growth Cones Are Not Required for Initial Establishment of Polarity or Differential Axon Branch Growth in Cultured Hippocampal Neurons
}

\author{
Gordon Ruthel and Peter J. Hollenbeck \\ Department of Biological Sciences, Purdue University, West Lafayette, Indiana 47907
}

Hippocampal neurons developing in culture exhibit two types of differential, seemingly competitive, process outgrowth in the absence of external cues. During the initial acquisition of polarity, one of several equivalent undifferentiated minor neurites preferentially grows to become the axon. Once the axon has formed, it typically branches, and the branches grow differentially rather than concurrently. In axons with only two branches, growth alternates between branches. In both axon establishment and branch growth alternation, growth among sibling processes or branches must be differentially regulated. We found that elaborate and dynamic growth cones were associated with growth, whereas diminished growth cones were associated with nongrowing processes or branches. To test whether growth cones were necessary for differential growth, growth cone motility was eliminated by application of cytochalasin E. Although cytochalasin treatment before axon formation yielded longer processes overall, a similar percentage of both treated and untreated neurons had one process that grew more rapidly and became much longer than its sibling processes. Immunostaining to visualize dephospho-tau, an axonal marker, demonstrated that these single dominant processes were axons. Axons that formed in cytochalasin were thicker and showed more intense anti-tubulin staining than their sibling processes. Branched axons deprived of growth cones retained a pattern of differential growth and often included alternation. These results indicate that neither formation of a single axon nor differential growth of branches are dependent on growth cone motility and suggest that the neuron can regulate neurite elongation at sites other than at the growth cone.

Key words: growth cone; axon outgrowth; cytochalasin; polarity; cell culture; sibling bias
During the development of neuronal connections, a neuron must generate a precise morphology that will support its specific function within the nervous system. For many neurons, this means that the development of several immature neurites must be regulated to form just one axon, and subsequently the branches of that axon must be differentially regulated so that certain branches will elongate, whereas others are eliminated (O'Leary et al., 1990). In some neuronal cell types, the outgrowth of each neurite appears to be regulated independently from its sibling processes and is controlled by the advance of the growth cone. For example, in cultures of chick sensory neurons, single cell bodies give rise to multiple axons and axonal branches that show simultaneous, rapid, and consistent growth (Lamoureux et al., 1998). However, preferential growth of one process or branch in other neuronal cell types has been suggested to result from competition for cellular resources (Smalheiser and Crain, 1984; Goslin and Banker, 1989). The hypothesis that differential process outgrowth is regulated by preferential transport of essential materials into a particular process has been termed "sibling bias."

Evidence suggesting competitive outgrow th among sibling processes in vivo has been obtained in neurons of the leech (Gan and Macagno, 1997), the cricket (Murphey and Lemere, 1984), and

Received Nov. 10, 1999; revised Dec. 21, 1999; accepted Jan. 5, 2000.

This work was supported by National Institutes of Health Grant NS27073. G.R. was supported in part by National Institutes of Health Training Grant 2 T32 NS07009-21 and a fellowship from the Harvard Mahoney Neuroscience Institute. We thank Dr. Peggy Criswell for comments on this manuscript.

Correspondence should be addressed to Dr. Peter Hollenbeck, Department of Biological Sciences, Lilly Hall, Purdue University, West Lafayette, IN 47907. E-mail: phollenb@purdue.edu.

Copyright (C) 2000 Society for Neuroscience $0270-6474 / 00 / 202266-09 \$ 15.00 / 0$ the hamster (Schneider, 1973). In bifurcated axons of cultured Aplysia neurons, appropriate target neurons placed at the end of one growing branch cause suppression of both growth and organelle transport in the other branch (Goldberg and Schacher, 1987).

Apparent competition among processes occurs in the absence of external cues in cultured rat hippocampal neurons, which typically form only a single axon from among several equivalent undifferentiated neurites (Dotti et al., 1988). In addition, studies of this system (Futerman and Banker, 1996; Ruthel and Banker, 1999) have suggested that the branches of individual axons do not grow in unison but rather alternate their growth in a manner consistent with the sibling bias hypothesis.

Do neurons regulate the preferential growth of one neurite or branch by preferential sorting of transported materials or is differential growth primarily under the control of the growth cone? Growth cones are well known to integrate extracellular information and to regulate axon outgrowth (Stoeckli, 1997). Rapid long-distance signaling between growth cones of an individual neuron has been reported previously (Zheng et al., 1994), suggesting that growth cones themselves might orchestrate the seemingly competitive differential growth of processes or branches. Furthermore, it has been suggested that the choice of a process to become an axon is linked to the formation of a larger and more dynamic growth cone on that process, either as a result of increased membranous organelle transport into that process (Bradke and Dotti, 1997) or as the initial, causative event specifying axonal character (Bradke and Dotti, 1999).

Our observations suggested that actively growing processes or branches possessed larger, more highly motile growth cones. We 

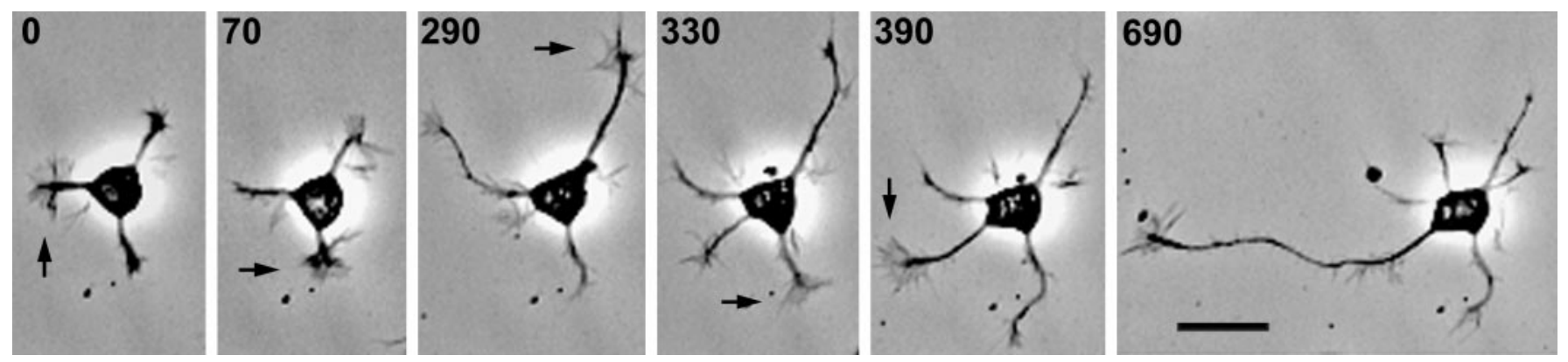

Figure 1. Axon determination: the transition of a neuron from stage 2 to stage 3 . The undifferentiated minor processes of a stage 2 neuron exhibit net growth over time as each process undergoes cycles of growth and retraction. Growth cone size reflects the dynamic changes in the growth states of the different processes, becoming large in actively growing processes (arrows). Eventually, one process grows longer than the others and continues to grow rapidly, differentiating into an axon. Relative times are shown in minutes, beginning at $\sim 8 \mathrm{hr}$ in culture. Scale bar, $20 \mu \mathrm{m}$.

therefore tested whether differences in growth cone size and motility were required for hippocampal neurons to initially establish a single axon or subsequently regulate the alternation of axonal branch growth. Because the structure of the growth cone, as well as its ability to control the rate and direction of outgrowth, is believed to be dependent on its actin filaments (Lin et al., 1994), we used cytochalasin E (CE) to disrupt actin filaments. Previous studies have shown that elongation of the axon can still occur when the growth cone is eliminated by treatment with cytochalasin (Marsh and Letourneau, 1984; Bentley and ToroianRaymond, 1986; Morris and Hollenbeck, 1993, 1995). Our results indicate that selection of a single minor process to become an axon and differential growth of separate axonal branches can occur in the absence of growth cones.

\section{MATERIALS AND METHODS}

Cell culture. Hippocampal neurons were cultured from age embryonic day 18 rats as described previously (Goslin and Banker, 1991) with modification. In brief, isolated hippocampi were dissociated with trypsin, followed by trituration with Pasteur pipettes. Neurons were plated onto poly-L-lysine-coated glass coverslips. After attachment of the cells to the substrate $(\sim 4 \mathrm{hr})$, coverslips were transferred to dishes containing confluent monolayers of glia, with neurons separated from glia by wax dots applied to the coverslip. Two significant modifications were made to the published protocol. $\mathrm{CO}_{2}$-independent medium (Life Technologies, Grand Island, NY) supplemented with $2 \%$ glucose, $1 \mathrm{~mm}$ pyruvate, $2 \mathrm{~mm}$ glutamine, $0.1 \%$ ovalbumin, and the N2 supplements of Bottenstein and Sato (1979) was used in place of Minimum Essential Medium (MEM) to maintain the neurons after transferal to dishes with glia. This modification was made to avoid fluctuations in $\mathrm{pH}$ that occur when bicarbonatebuffered mediums are exposed to air and is particularly notable because of its readily apparent superiority over HEPES-buffered mediums for maintaining cell health in non- $\mathrm{CO}_{2}$ enriched environments. A second modification was made to extend the usefulness of cells obtained from each dissection. Because only cells in the first $2 \mathrm{~d}$ after plating were used for this study, aliquots of cells from each dissection were cryogenically preserved at $-80^{\circ} \mathrm{C}$ in MEM with $10 \%$ horse serum and $8 \%$ dimethylsulfoxide (DMSO) and then thawed and plated at later times. As observed previously (Mattson and Kater, 1988), cryopreserved neurons were indistinguishable from neurons plated immediately after dissociation. Cells were used at 1-2 d in culture for branch growth studies and beginning at $\sim 8 \mathrm{hr}$ in culture for axon establishment studies.

Image acquisition and analysis. Coverslips bearing neurons were secured into culture chambers and placed on the heated $\left(34-36^{\circ} \mathrm{C}\right)$ stage of a Nikon (Tokyo, Japan) inverted microscope. Culture chambers consisted of a neuron-bearing coverslip sealed to a second coverslip separated from the first by a Teflon spacing ring, all held in an aluminum retainer. The space between the coverslips was filled with glialconditioned culture medium $(\sim 0.5 \mathrm{ml})$. A different type of chamber was used for cytochalasin application. This chamber consisted of an aluminum bottom piece that held the coverslip with neurons, a Teflon ring, and a Teflon top piece with a central well filled with culture medium $(\sim 1.5$ $\mathrm{ml})$. An easily removable coverslip was sealed over the top of the Teflon well with vacuum grease to prevent evaporation but to allow access for exchange of medium. Images were acquired at intervals using a Hamamatsu cooled CCD camera and Metamorph imaging software (Universal Imaging, West Chester, PA). In many cases, a Ludl motorized microscope stage was used to follow multiple neurons over the same recording period.

Length measurements were made using a calibrated measure function of the Metamorph software and graphed using SigmaPlot software (SPSS Inc., Chicago, IL). For statistical analysis of branch growth, cells having only two axonal branches were chosen, and the branches were randomly designated as branch 1 and branch 2 . For every branch 1, periods of growth and nongrowth were identified according to strict criteria. Average outgrowth rates were required to be $>7$ or $<4 \mu \mathrm{m} / \mathrm{hr}$, respectively, and continuing for at least $50 \mathrm{~min}$ until a break in trend of $30 \mathrm{~min}$ or more. For CE-treated cells, the restriction that time points were $>10 \mathrm{~min}$ after CE application was added to the above criteria. Average growth rates were calculated for these periods in each branch 1 and for the corresponding time period in each sibling branch 2. Paired $t$ tests (or nonparametric equivalents as dictated by the normality of the data distribution) were performed separately for rates obtained for defined growing and nongrowing periods with their respective sibling pairs. $t$ tests (or nonparametric equivalent) were performed for the two resulting sets of branch 2 rates.

Cytochalasin treatment. Cytochalasin E (Sigma, St. Louis, MO) was used to disrupt actin filaments, thereby eliminating growth cone motility. This variety of cytochalasin was chosen for its apparent lack of side effects and previous success in long-term treatment of cultured neurons (Morris and Hollenbeck, 1995). Medium bathing neurons was replaced with glial-conditioned medium containing $0.17-0.23 \mu \mathrm{g} / \mathrm{ml} \mathrm{CE}$ made from a stock solution of $0.2 \mathrm{mg} / \mathrm{ml} \mathrm{CE}$ in DMSO.

Immunocytochemistry. Cells were prepared for staining by fixing with a $4 \%$ paraformaldehyde and $4 \%$ sucrose solution for $15-20 \mathrm{~min}$, rinsing twice for $1 \mathrm{~min}$ in PBS, and permeabilizing with $0.2 \%$ Triton $\mathrm{X}-100$ for $10 \mathrm{~min}$. After three rinses for $2 \mathrm{~min}$ in PBS, cells were incubated with $10 \%$ bovine serum albumin for $1 \mathrm{hr}$ at $37^{\circ} \mathrm{C}$ and then overnight at $4^{\circ} \mathrm{C}$ with 1:400 tau-1 anti-dephospho-tau (Boehringer Mannheim, Indianapolis, IN) or 1:400 DM1A anti-tubulin (Amersham, Arlington Heights, IL). After three PBS rinses of $15 \mathrm{~min}$ each, cells were incubated with 1:400 fluorescein or CY-2-conjugated secondary antibody and Texas Red phalloidin (Molecular Probes, Eugene, OR) for $1 \mathrm{hr}$ at $37^{\circ} \mathrm{C}$. After three more 15 min PBS rinses and $1 \mathrm{~min}$ in distilled water, coverslips were mounted onto microscope slides for viewing.

\section{RESULTS}

\section{Minor processes compete for axonal character}

As reported previously (Dotti et al., 1988; Goslin and Banker, 1989), we found that undifferentiated minor processes go through unsynchronized extensions and retractions until one process becomes longer and continues to grow, taking on typical axonal character (Fig. 1). It has been reported that the growth cone of the minor process that will become the axon becomes much larger than the growth cones of the other processes (Bradke and Dotti, 1997, 1999). However, by following cells with time-lapse video 
microscopy over many hours before axon formation, we have found that each of the several processes of a neuron can have the largest growth cone at different times before elaboration of a single axon (Fig. 1, arrows). Larger growth cones tended to be associated with periods of elongation in any of the processes, not only when that elongation was sustained during differentiation into an axon. Although this greatly limits the usefulness of growth cone size as a predictor of axon determination, the association between growth cone motility and elongation nonetheless raises the question of whether growth cones mediate the establishment of an axon.

\section{Competition for axonal character is not dependent on growth cones}

To test whether growth cone motility was essential for the establishment of only a single axon, we eliminated growth cones by disrupting the actin filaments that are required for both the maintenance of growth cone morphology and for the regulation of the rate and direction of outgrowth by the growth cone (Lin et al., 1994). This was accomplished by treating cells that had not yet formed an axon with cytochalasin $\mathrm{E}$, a variety of cytochalasin believed to be free of cellular effects other than its inhibition of actin polymer assembly. This variety has also been shown to be effective for long-term treatment of cultured neurons without compromising cell survival (Morris and Hollenbeck, 1995). Doses in the range of $0.17-0.23 \mu \mathrm{g} / \mathrm{ml} \mathrm{CE}$ were used. This concentration resulted in a complete loss of lamellipodial and filopodial activity, often leaving a static membranous remnant. The distal portion of elongating processes were occasionally observed to lift temporarily off of the substrate (Figs. 2, 3), an event never observed when even the smallest of growth cones is present, providing further indication that the growth cones were eliminated by this treatment. We also confirmed that this dose does not inhibit the outgrowth of established axons. In fact, axon outgrowth rates were found to be significantly increased in the presence of CE $[23.5 \pm 2.7 \mu \mathrm{m} / \mathrm{hr}(\mathrm{SEM})$ compared with $6.8 \pm$ $1.3 \mu \mathrm{m} / \mathrm{hr}$ (SEM) for untreated axons; $n=6$ for each condition; $p=0.002$ using a Mann-Whitney rank sum test]. Higher doses were not used because, when tested with established axons, they yielded increasingly undirected and tangled outgrowth that impeded accurate measurement.

Cells at both stage 1 (lamellipodia but no processes) and stage 2 (several minor processes but no axon) were chosen for study. Stage 1 cells were able to form processes in the presence of $\mathrm{CE}$ (Figs. 3, 4), and these processes were still capable of forming branches in the absence of growth cones (Fig. 2). Although CE treatment typically yielded overall longer and thinner processes, both cells with and without minor processes at the onset of treatment frequently formed a single dominant (both longer and thicker) process in CE (Fig. 2). Table 1 shows the percentages of neurons forming one axon (defined as a process at least twice as long as any other process of the same cell and with a minimum length of $50 \mu \mathrm{m}$ ), two axons (two processes of similar length each at least $80 \mu \mathrm{m}$ long and twice as long as the remaining processes), or no axon (all similar length processes regardless of absolute length) over a recording period of $14 \mathrm{hr}$ that began at $8 \mathrm{hr}$ in culture. Percentages were calculated separately for cells initially at stage 1 or at stage 2 for both untreated and CE-treated neurons.

The percent of cells meeting the criteria for a single axon were similar regardless of whether the cells were treated with $\mathrm{CE}$ or untreated. The fraction of hippocampal neurons forming an axon
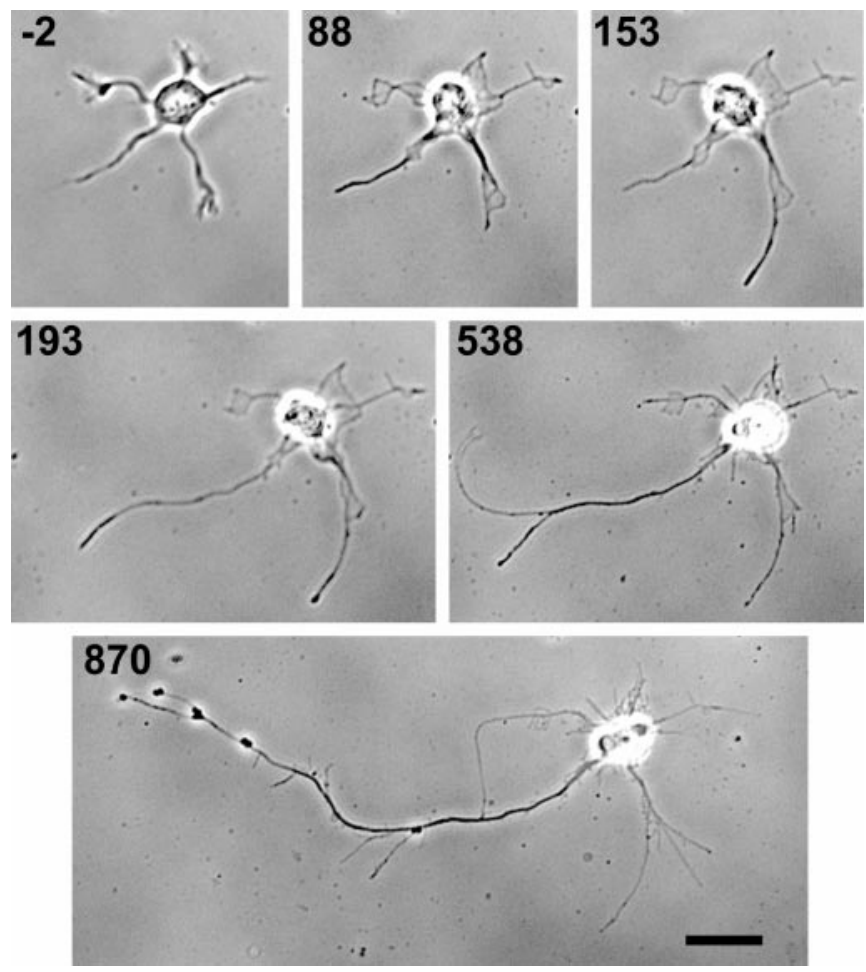

Figure 2. Formation of an axon in the presence of $0.2 \mu \mathrm{g} / \mathrm{ml}$ cytochalasin E. A stage 2 neuron is shown before addition of $\mathrm{CE}$ and at time intervals thereafter. Growth cones are eliminated by treatment with $\mathrm{CE}$, but processes nonetheless elongate. The elimination of growth cone function is clearly demonstrated by occasional lifting of the process tip off the substrate. Although substantial growth is seen in several of the processes, one process becomes significantly longer and also takes on an apparently greater thickness compared with the other processes. Branching in the absence of growth cone formation was also noted to occur. Times are shown in minutes relative to the time of CE addition. Scale bar, $20 \mu \mathrm{m}$.

Table 1. Percentages of neurons forming one, two, or no axons

\begin{tabular}{lrrrr} 
Initial stage & No axon & One axon & Two axons & Total \\
\hline Control & & & & \\
1 & $16(64 \%)$ & $9(36 \%)$ & $0(0 \%)$ & 25 \\
2 & $52(46 \%)$ & $57(50 \%)$ & $5(4 \%)$ & 114 \\
CE-Treated & & & & \\
1 & $54(51 \%)$ & $46(44 \%)$ & $5(5 \%)$ & 105 \\
2 & $43(37 \%)$ & $57(49 \%)$ & $16(14 \%)$ & 116 \\
\hline
\end{tabular}

Percentages of neurons that formed one, two, or no axons under control conditions versus cytochalasin $E$ treatment over a $14 \mathrm{hr}$ recording period starting at $8 \mathrm{hr}$ in culture. Data are further subdivided into neurons observed beginning at stage 1 (lamellipodial fringe but no neurites) and stage 2 (minor neurites only). See Results for criteria used to categorize neurons.

by $24 \mathrm{hr}$ in culture has been reported previously to be $<40 \%$ (Jareb and Banker, 1997). Our percentages are higher, probably because our sample population consisted of cells that had already attained typical stage 1 or stage 2 morphology by $8 \mathrm{hr}$ in culture. We found a substantially higher incidence of neurons that met the criteria for having two axons in CE compared with untreated neurons, with correspondingly fewer cells that had processes all of similar length (Table 1).

To confirm that single axons were established in CE, we selected neurons before $\mathrm{CE}$ treatment and followed them continuously so that they were known to have formed the presumptive 

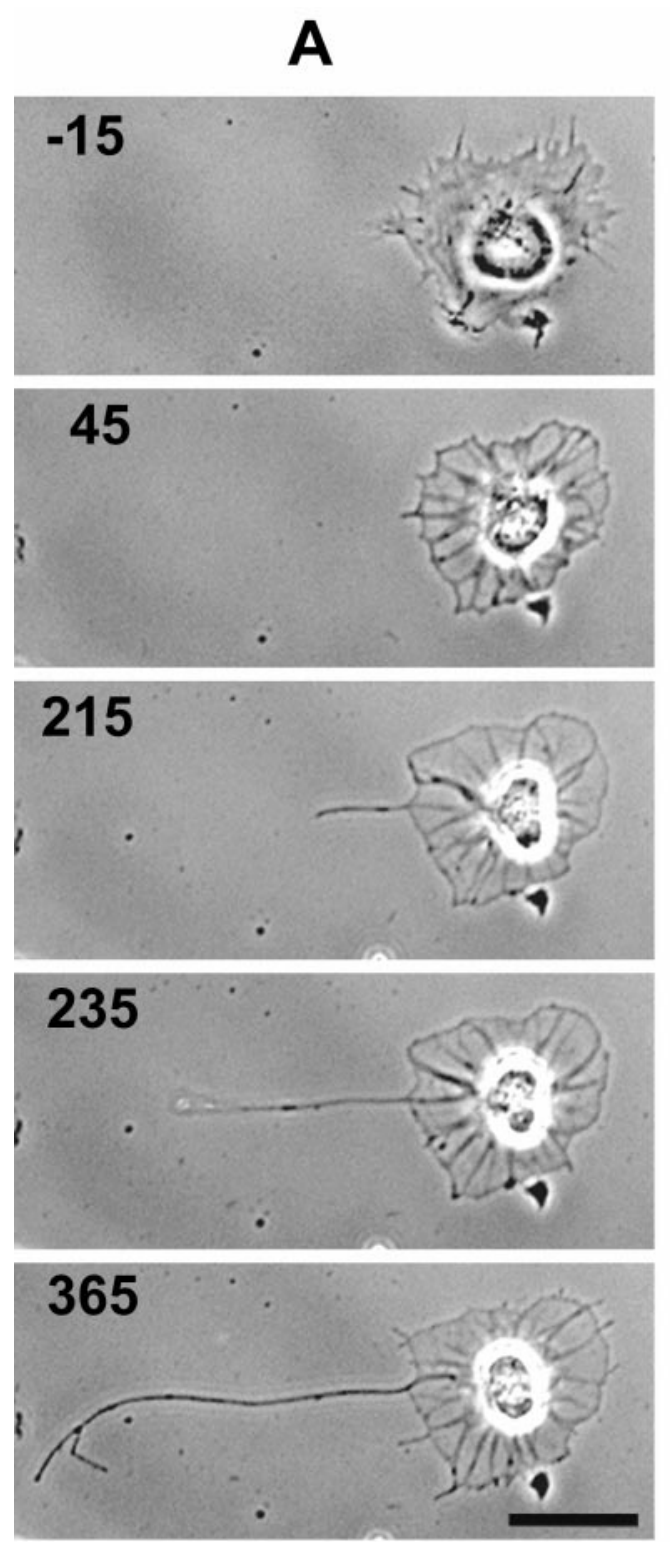

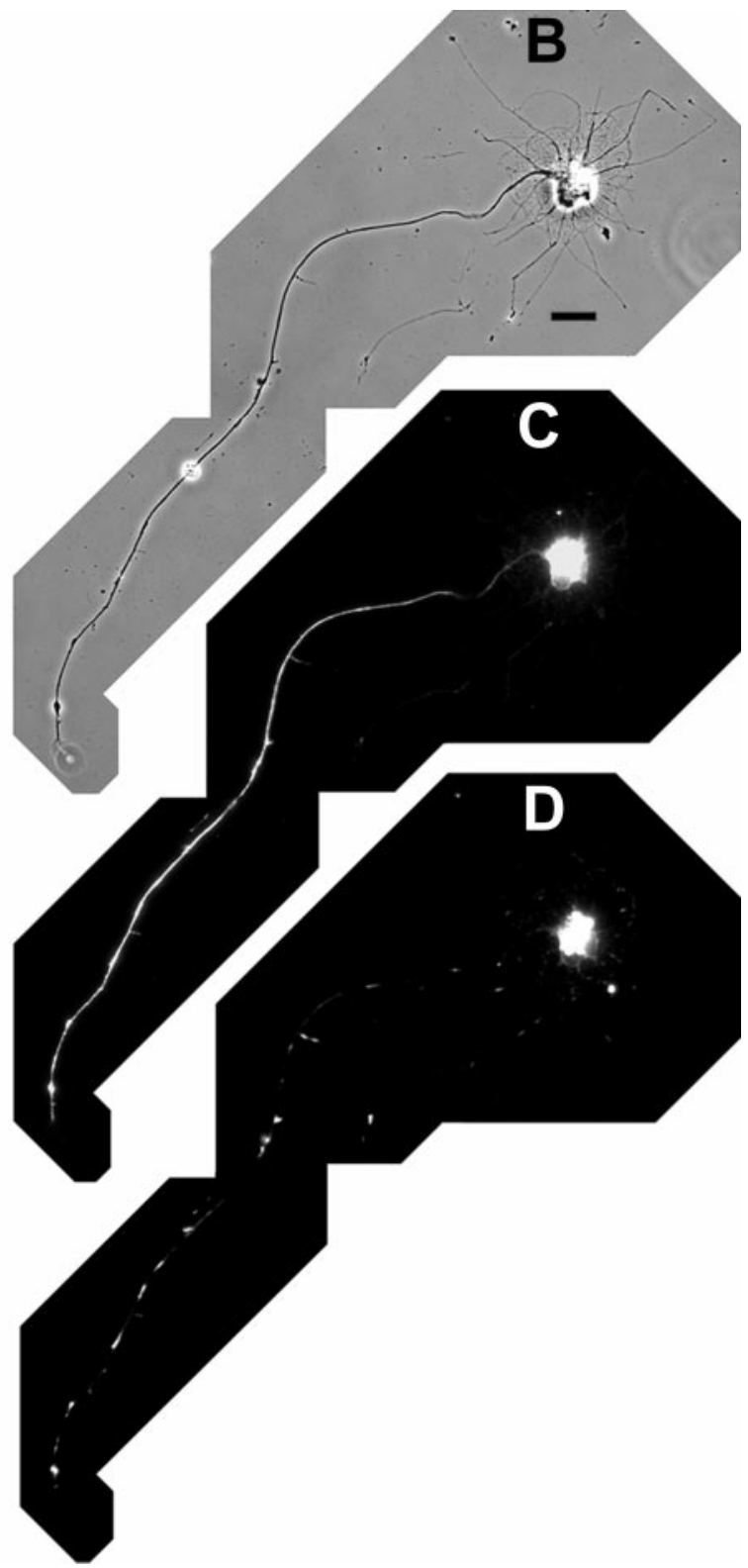

Figure 3. Tau-1 and Texas Red phalloidin staining of an axon that formed in the presence of cytochalasin E. $A$, An axon forms from a stage 1 neuron treated with $0.17 \mu \mathrm{g} / \mathrm{ml} \mathrm{CE}$. The cell exhibits the typical actin-based lamellipodial fringe characteristic of stage 1 neurons before addition of CE. A long process grows from the remnant of the lamellipodial fringe after CE is applied. The image taken at 235 min after addition of CE shows the process tip lifting off the substrate, underscoring the effectiveness of the $\mathrm{CE}$ application in eliminating growth cones. Times are shown in minutes relative to the time of $\mathrm{CE}$ addition. $B$, The same cell shown in $A$ was fixed $\sim 16 \mathrm{hr}$ after $\mathrm{CE}$ addition and double-stained with tau-1 antibody $(C)$ and Texas Red phalloidin $(D)$. Tau-1 specifically stained the longest process in a distal to proximal gradient. Phalloidin staining shows aggregates of F-actin in the cell body and along the processes, confirming the effectiveness of the CE. Scale bars, $20 \mu \mathrm{m}$.

axon while in CE. After these neurons had formed a single dominant process, they were stained with the tau- 1 antibody to dephospho-tau, which specifically stains axons in a distal to proximal gradient (Mandell and Banker, 1996). As shown in Figure 3, tau-1 staining was segregated to the single longest process and was brighter distally, consistent with the identity of this process as the axon. Because the axons that formed in CE appeared to be thicker than the other processes and because it has been reported previously that axons contain more microtubules per unit length than minor processes ( $\mathrm{Yu}$ and Baas, 1994), we wished to test whether the thickness we observed might be partly attributable to a difference in microtubule mass. We therefore identified neurons that were known to have formed their axon in $\mathrm{CE}$ and stained them with DM1A, an antibody to $\alpha$-tubulin. As shown in Figure 4 , the presumptive axon showed much brighter tubulin staining than did the other processes. Staining with Texas Red phalloidin to visualize actin filaments showed aggregates of F-actin in the cell body and along the processes, verifying the effectiveness of CE treatment (Figs. 3D, 4D).

\section{Hippocampal axons exhibit branch growth alternation}

Once one process has become an axon, another form of apparent competition for growth takes place. The branches of cultured hippocampal axons most often do not grow simultaneously, but 

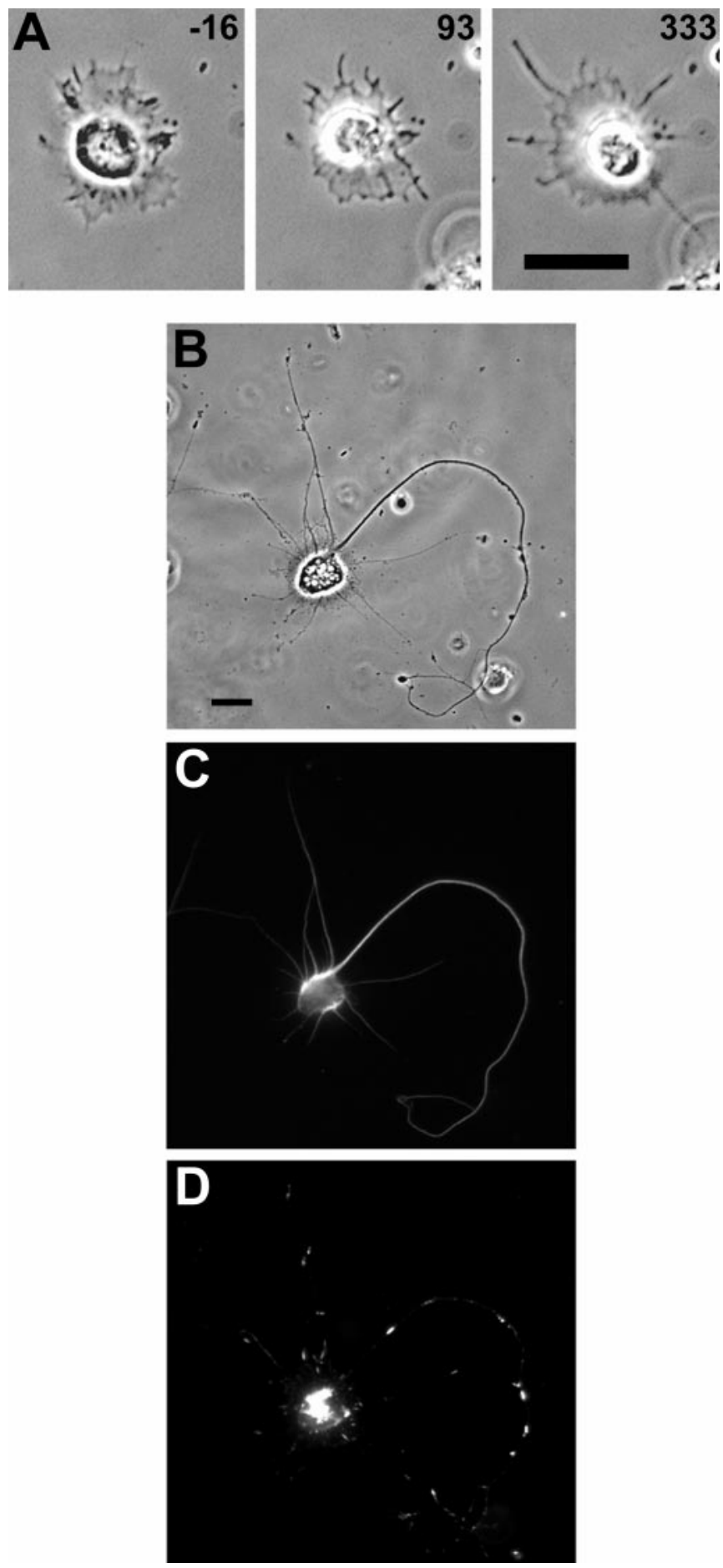

Figure 4. DM1A anti-tubulin and Texas Red phalloidin staining of an axon that formed in the presence of cytochalasin E. $A$, A stage 1 neuron is shown before and after addition of $0.2 \mu \mathrm{g} / \mathrm{ml} \mathrm{CE}$. After CE is added, lamellipodial activity ceases, leaving behind a membranous remnant. Processes grow out of the lamellipodial remnant. Times are shown in minutes relative to the time of $\mathrm{CE}$ addition. $B, \mathrm{By} \sim 16 \mathrm{hr}$ in $\mathrm{CE}$, the cell has extended multiple long processes, with one of these processes at least twice as long as any of the other processes and also thicker. $C$, The cell was fixed and stained with DM1A, an antibody to tubulin. A greater degree of staining in the longest process (the presumptive axon) suggests that the apparently greater thickness of this process is at least in part attributable to a higher amount of tubulin. $D$, Phalloidin staining shows a staining pattern indicative of F-actin aggregates in the cell body and along the processes. Scale bars, $20 \mu \mathrm{m}$. rather, as shown in the example of Figure 5, alternate their growth such that only one branch is growing rapidly at any given time. As the graph (Fig. 5B) shows, periods of change between growing and nongrowing states in one branch correlate with the converse change in the other branch (Futerman and Banker, 1996, their Fig. 3). In axons with higher order branching, the growth states of the separate branches likewise showed an apparent interdependence, but the association among growth states of different branches was more complex than the simple alternation of growth observed between only two branches. We therefore limited our analysis in this study to axons with a single branch point.

First, quantitative analysis was used to demonstrate that alternation of branch growth is a consistent characteristic of untreated cultured hippocampal neurons. We assessed growth in separate branches of individual axons by measuring the average elongation rate of one branch of each axon during intervals when that branch exhibited substantial net growth (at least $7 \mu \mathrm{m} / \mathrm{hr}$ for a minimum of $50 \mathrm{~min}$; see Materials and Methods for detailed criteria). This rate was then compared with the rate of outgrowth in the sibling branch during the same time period. The comparison yielded a significant difference, with mean elongation rates of $17.9 \mu \mathrm{m} / \mathrm{hr}$ for growing branches and $-0.9 \mu \mathrm{m} / \mathrm{hr}$ for their sibling branches ( $n=12$ periods from 10 cells; $p<0.001$ using a paired $t$ test). Conversely, identification of intervals without substantial growth $(<4 \mu \mathrm{m} / \mathrm{hr}$ for a minimum of $50 \mathrm{~min}$; see Materials and Methods for detailed criteria) in one branch of each axon and comparison with growth rate in the sibling branch over the same time period likewise yielded a significant difference, with means of $-5.1 \mu \mathrm{m} / \mathrm{hr}$ for the nongrowing branch and $7.2 \mu \mathrm{m} / \mathrm{hr}$ for its sibling branch ( $n=14$ periods from 12 cells; $p<0.001$ using a paired $t$ test).

In addition, to ensure that there was truly a concomitant change in sibling branch growth rate as predicted by alternation of branch growth and that the differences above were not artificially created by selecting particularly high and low rates in the defined branch, we also compared the two average growth rates obtained for the sibling branch. We found a significant change in rate for the sibling branch that opposed the rate change in the defined branch ( $p<0.001$ using a Mann-Whitney rank sum test).

As can be seen in the phase contrast images in Figure $5 \mathrm{~A}$, growing branches tended to have elaborate and highly motile growth cones, whereas nongrowing branches tended to have relatively small growth cones with little protrusive activity. This observation in conjunction with the knowledge that outgrowth can be regulated at the growth cone (Lin et al., 1994; Stoeckli, 1997) and evidence that rapid signaling can occur between distant growth cones (Zheng et al., 1994) led us to ask whether the growth cones were responsible for orchestrating the alternation of growth between branches.

\section{Elimination of growth cones does not eliminate differential branch growth}

To test the hypothesis that growth cone motility determines differential branch growth, growth cones were again eliminated by the application of $\mathrm{CE}$, as described above. As illustrated by the example in Figure 6, differential growth was still observed in branched axons after elimination of growth cones with CE. In several cases, cells exhibited growth in only one branch during nearly the entire recording period, but alternation of branch growth was observed in many cells. Times immediately after CE application were often marked by either a rapid simultaneous growth or a temporary cessation of growth in both branches 

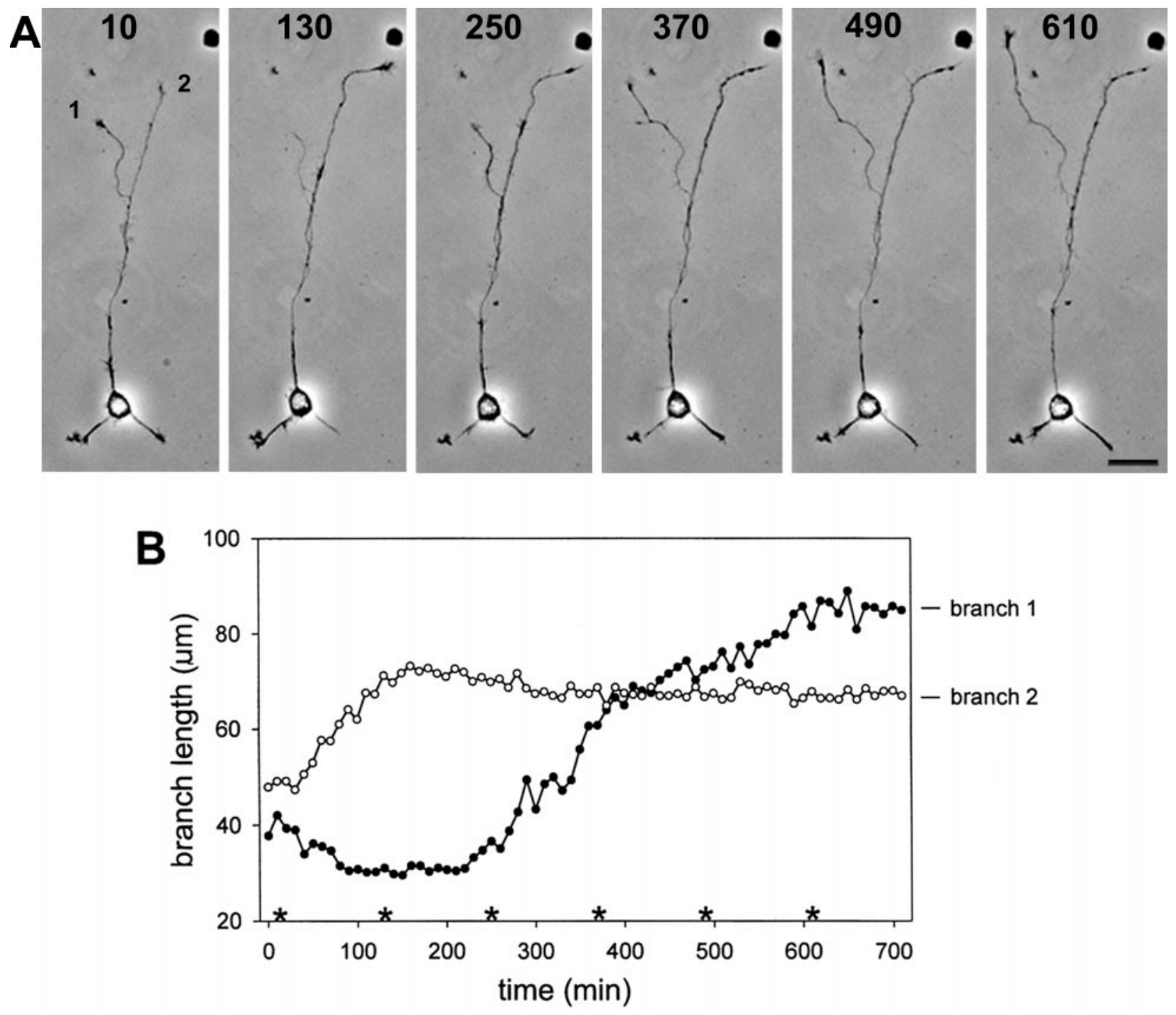

Figure 5. Alternation of axon branch growth. $A$, Phase contrast images of a neuron with a branched axon at selected time points. The two branches alternate their growth state such that only one branch grows at a time. Growth cone size reflects the growth state of the branch, with the growing branch exhibiting an elaborate and highly motile growth cone, whereas the growth cone of the nongrowing branch is relatively small and inactive. Branches are labeled as 1 and 2 in reference to the graph in $B$. Relative times are shown in minutes and refer to the times in the graph in $B$. Scale bar, $20 \mu \mathrm{m}$. $B$, Branch length (measured from the branch point) is graphed as a function of time for the cell shown in $A$. Branch 1 was growing at the onset of recording, branch 2 was not. Soon after recording commenced, the branches switched growth states. Growth alternated once more at $\sim 200$ min of recording. Asterisks mark times that correspond to the images shown in $A$.

before a pattern of differential growth was re-established. Differential branch growth in the presence of CE was evaluated statistically as described above for untreated branches. Growth rates during periods meeting the criteria for substantial growth were significantly higher than rates in the sibling branches during the same time periods (medians of 23.2 and $4.5 \mu \mathrm{m} / \mathrm{hr}$, respectively; $n=18$ periods from 12 cells; $p=0.002$ using a Wilcoxon signed rank test). Rates during defined periods of nongrowth were likewise significantly lower than growth rates of the sibling branches in the matching time periods (means of -0.6 and 14.8 $\mu \mathrm{m} / \mathrm{hr}$, respectively; $n=25$ periods from 15 cells; $p<0.001$ using a paired $t$ test). To demonstrate that growth rate in the sibling branch underwent a change to complement the periods of growth and nongrowth in the defined branch, the two sets of growth rates from the sibling branch were compared and found to be significantly different ( $p=0.047$ using the Mann-Whitney rank sum test). Together, these tests clearly confirm that alternating branch growth continued after growth cones were eliminated by treatment with CE.

\section{DISCUSSION}

We have examined the role of growth cone motility in determining differential growth states among sibling neurites and between sibling branches. By eliminating growth cones with cytochalasin E, we determined that growth cones are not required for the coordination of growth that results in the formation of a single axon from among several processes that each have the potential to become an axon (Goslin and Banker, 1989). Although all processes grew longer when growth cones were eliminated with $\mathrm{CE}$ than in untreated cells, in many cases one process clearly emerged as significantly longer, faster growing, and axon-like. The axonal character of the single longer process was supported by staining 

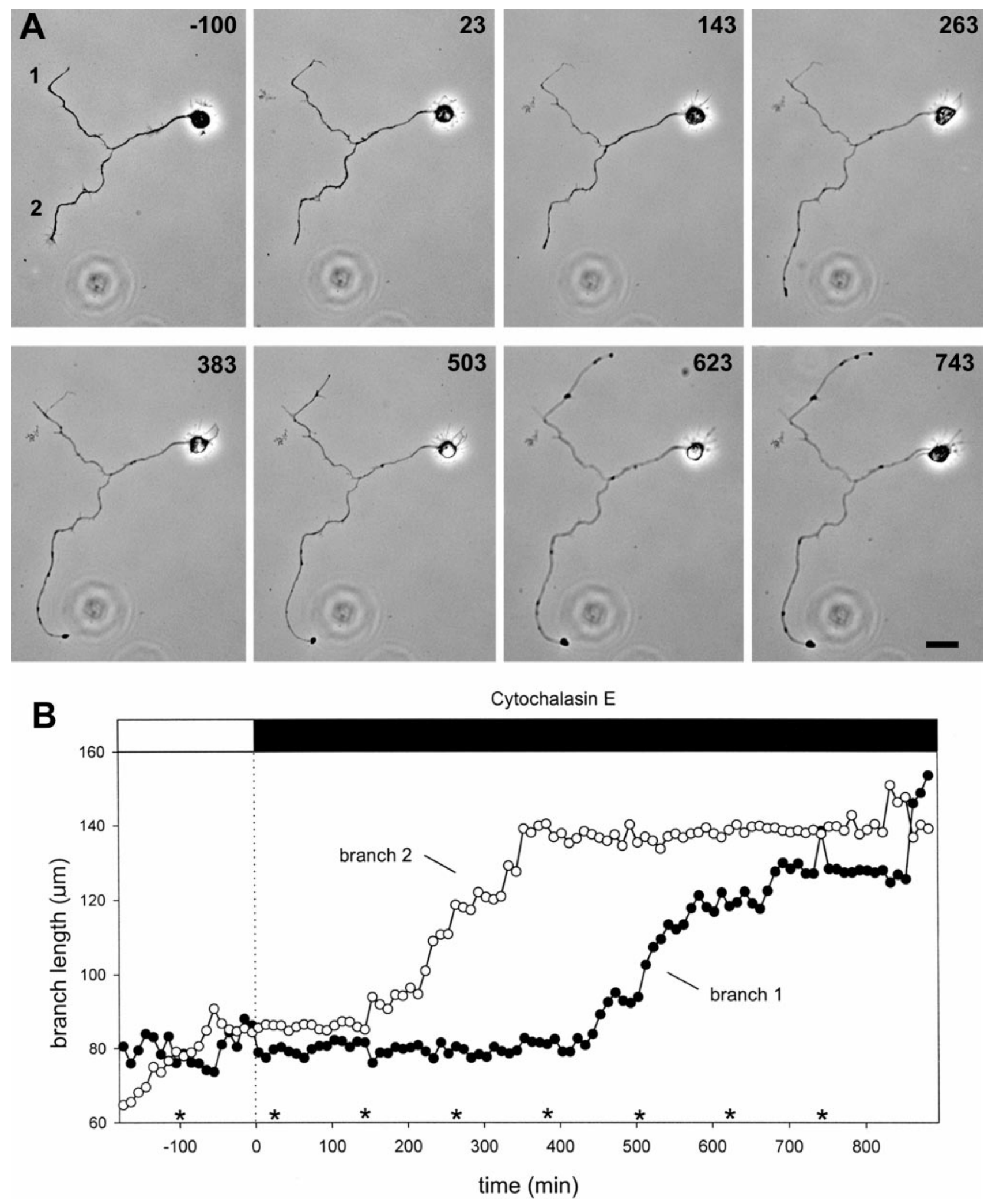

Figure 6. Differential axon branch growth in the presence of cytochalasin E. $A$, The branched axon of a neuron is shown before and after addition of $0.17 \mu \mathrm{g} / \mathrm{ml}$ CE. Growth cones are eliminated by the treatment with CE, but growth of the branches nonetheless proceeds. Growth of the branches continues to occur one branch at a time. Branches are marked as 1 and 2 in reference to the graph in $B$. Times are shown in minutes relative to the time of CE addition. Scale bar, $20 \mu \mathrm{m}$. B, Branch length (measured from the branch point) is graphed as a function of time. Before cytochalasin (open bar at $t o p$ ), the branches show differential growth that alternates at approximately $-50 \mathrm{~min}$. After addition of $\mathrm{CE}($ dotted line, time $=0)$, both branches cease growing for $\sim 2 \mathrm{hr}$. In the continued presence of CE ( filled bar at top), branch 2 begins to grow after $\sim 150$ min in CE, whereas branch 1 remains nongrowing. At $\sim 380 \mathrm{~min}$ in CE, the branches alternate their growth state such that growth ceases in branch 2 and begins in branch 1 . Asterisks mark times that correspond to phase contrast images in $A$. 
with antibody to dephospho-tau, an axonal marker (Mandell and Banker, 1996), and by increased anti-tubulin staining that suggested that the longer process contained a greater number of microtubules than the remaining processes (Yu and Baas, 1994). We have demonstrated that the branches of cultured hippocampal axons undergo alternation of growth, one branch pausing as the other undergoes an extended period of average growth, suggesting that the growth states of the branches are interdependent. Although it has been documented that actions at one process tip can have effects on growth at another distant process tip (Goldberg and Schacher, 1987; Zheng et al., 1994), the alternation of branch growth in this system occurs in the apparent absence of external cues, suggesting that coordination of growth in different branches of an axon can be intrinsically determined. Although in control cells we found concomitant changes in growth cone size and motility with changes in branch growth state, when we eliminated growth cones with $\mathrm{CE}$, branches still exhibited differential growth. This suggests that branch growth may at least partially be controlled more proximally, perhaps at the branch point. Together, these results indicate that differences in growth cone motility are not the primary means of determining differential growth among separate processes of the same cell or between different branches of an individual axon.

\section{The role of the growth cone}

Despite a correlation between larger growth cones and more rapid outgrowth in our cultures, we found that growth cone motility is not essential for determining which process or branch will grow more rapidly. Although we cannot discount the involvement of growth cone functions not dependent on intact actin filaments that may have remained unaffected by $\mathrm{CE}$ treatment, the ability of the growth cone to regulate both the rate and direction of outgrowth is believed to be actin-dependent (Lin et al., 1994). Indeed, we observed increases in minor process length and rate of axon outgrowth in the presence of $\mathrm{CE}$, suggesting that growth cone regulation of outgrowth was affected. It is plausible that growth cones act to limit process outgrowth in hippocampal neurons as suggested previously (Bradke and Dotti, 1999). This is consistent with findings showing that disruption of actin filaments leads to microtubule invasion into the peripheral domain of Aplysia growth cones from which they are otherwise primarily excluded (Forscher and Smith, 1988).

The association of elaborate growth cones with growing processes in neuronal cultures is particularly curious in light of studies of in vivo axon outgrowth that found rapidly elongating axons had growth cones of relatively simple morphology, whereas axons that paused at directional decision points possessed the most elaborate growth cones (Tosney and Landmesser, 1985; Bovolenta and Mason, 1987). Because the cultured growth cones are moving along a uniform, nonphysiological substrate of polyL-lysine, they might be expected to mimic the situation at decision points in which sampling of the environment is crucial before determination of a direction. The continued capacity for differential branch growth in the absence of growth cones suggests that the changes in growth cone size observed to coincide with changes in branch growth state may result from other changes in a process that affect its capacity for growth, such as changes in transport capabilities or microtubule dynamics.

It should be noted that, although our results indicate that growth cones are not necessary for differential regulation of neurite outgrowth in a uniform environment, growth cones may nonetheless be required to mediate neurotrophic or other envi- ronmental control of selective outgrowth and elimination, as proposed previously (Crutcher and Saffran, 1990).

\section{Axon determination}

Although it has been reported (Bradke and Dotti, 1999) that elimination of growth cones with cytochalasin D or latrunculin B in stage 2 neurons resulted in multiple axons, axons were defined only as processes greater than $40 \mu \mathrm{m}$ in length with outgrowth rates that matched untreated axon growth rates. We have found that the outgrowth rates of established axons are greatly enhanced in $\mathrm{CE}$, suggesting that all processes show increased rates of elongation. Thus, in the previous study, processes of true axonal character might have been expected to show much higher growth rates than the control axons. It is therefore possible that the neurons in the previous study had not yet formed an axon but would have formed one under extended observation. Our findings indicate that axon determination can occur independent of the growth cone.

If differences in growth cone size and motility do not determine which process becomes an axon, then what are the other possibilities? Preferential sorting of organelles into the nascent axon has been reported (Bradke and Dotti, 1997); however, the temporal relationship with axon determination is unclear. Mitochondria cluster at the base of nascent axons (Bradke and Dotti, 1997; Mattson and Partin, 1999); however, this may be a consequence of increased metabolic activity in the more rapidly growing nascent axon because mitochondria are known to accumulate in regions of high energy consumption (Morris and Hollenbeck, 1993). Higher microtubule mass compared with minor processes has been reported as a characteristic of new axons ( $\mathrm{Yu}$ and Baas, 1994) and is consistent with the results of tubulin immunocytochemistry in CE-treated neurons in the current study. Because transport occurs along microtubules, this might in turn result in the aforementioned increase in organelle traffic. Higher microtubule content may itself result from a selective dephosphorylation of the microtubule-associated protein tau in the nascent axon. Blocking of tau dephosphorylation via the phosphatase inhibitor pervanadate (Mandell and Banker, 1998) or somewhat more selectively via inhibition of calcineurin (Ferreira et al., 1993) resulted in the inhibition of axon formation. However, it is conceivable that axon elongation was inhibited in those studies without necessarily affecting less obvious steps in the acquisition of polarity. Although it is unclear exactly how the determination of an axon occurs in hippocampal neurons, the choice of which minor neurite becomes the axon is unlikely to be predetermined because when an established axon is transected to the same length as the other processes, each neurite has an equal probability of becoming the new axon (Goslin and Banker, 1989).

\section{Differential branch growth}

Our findings indicate that, not only is there a competition among minor processes that allows one to become the axon, but there is also an intrinsically determined competition for growth among separate branches of an individual axon. The finding that differential branch growth still occurs after the elimination of growth cones by treatment with $\mathrm{CE}$ suggests that changes in branch growth state are not entirely under the control of the growth cone. Thus, there are likely to be differences between branches, other than in the size and motility of the growth cone, that affect the capacity for elongation.

If growth cones are not responsible for coordinating growth among different branches, then what are the other possibilities? 
Changes in branch growth state may reflect underlying changes in sorting of transported materials at the branch point (Goldberg and Schacher, 1987; Ruthel and Hollenbeck, 1998). At least in the case of mitochondria, changes in sorting to branches do occur in hippocampal cultures but appear to be subsequent to changes in growth state (Ruthel and Hollenbeck, 1998). It has been shown in lobster axons that vesicle transport occurs along only a small fraction of the microtubules (Miller et al., 1987). If the ability of a microtubule to act as a track for transport can be modulated in individual branches, then such modulation may form a basis for changes in sorting to different branches. Changes in microtubules or their associated proteins might likewise affect the ability of the microtubules to extend and thereby affect axon growth. Whatever changes might take place to determine branch growth, our findings suggest that these changes are localized to individual branches and are associated with reciprocal changes in other branches.

\section{Sibling bias?}

Both the establishment of a single axon and alternation of branch growth are consistent with the hypothesis of sibling bias. It is possible that preferential routing of materials into one process might favor growth in that process but limit growth where a supply of materials is not provided. Indeed, when growth conelike structures, which periodically form at the base of hippocampal axons and travel to the tip, are sorted into one branch, there is a subsequent burst of growth selectively in that branch (Ruthel and Banker, 1999). An alternative explanation is that long-range signaling from one process or branch to another might initiate changes that do not involve supplies of materials but nonetheless affect growth state. Numbers of autophagic vacuoles retrieving materials from the distal axon to the cell body for degradation were found to increase in sympathetic axons whose outgrowth was halted (Hollenbeck, 1993), indicating that a mechanism exists in neurons for removing unneeded materials that reach nongrowing tips. Regardless of the means by which the preferential growth of one process or branch is determined, our results indicate that growth cone motility is not an essential mediator of that determination.

\section{REFERENCES}

Bentley D, Toroian-Raymond A (1986) Disoriented pathfinding by pioneer neurone growth cones deprived of filopodia by cytochalasin treatment. Nature 323:712-715.

Bottenstein JE, Sato GH (1979) Growth rate of a neuroblastoma cell line in a serum-free supplemented medium. Proc Natl Acad Sci USA 96:514-519.

Bovolenta P, Mason C (1987) Growth cone morphology varies with position in the developing mouse visual pathway from retina to first targets. J Neurosci 7:1447-1460.

Bradke F, Dotti CG (1997) Neuronal polarity: vectorial cytoplasmic flow precedes axon formation. Neuron 19:1175-1186.

Bradke F, Dotti CG (1999) The role of local actin instability in axon formation. Science 283:1931-1934.

Crutcher KA, Saffran BN (1990) Developmental remodeling of neuronal projections: evidence for trophomorphism? Comments Dev Neurobiol 1:119-141.

Dotti CG, Sullivan CA, Banker GA (1988) The establishment of polarity by hippocampal neurons in culture. J Neurosci 8:1454-1468.

Ferreira A, Kinkaid R, Kosik KS (1993) Calcineurin is associated with the cytoskeleton of cultured neurons and has a role in the acquisition of polarity. Mol Biol Cell 4:1225-1238.

Forscher P, Smith SJ (1988) Actions of cytochalasins on the organization of actin filaments and microtubules in a neuronal growth cone. J Cell Biol 107:1505-1516.
Futerman AH, Banker GA (1996) The economics of neurite outgrowth - the addition of new membrane to growing axons. Trends Neurosci 19:144-149.

Gan WB, Macagno ER (1997) Competition among the axonal projections of an identified neuron contributes to the retraction of some of those projections. J Neurosci 17:4293-4301.

Goldberg DJ, Schacher S (1987) Differential growth of the branches of a regenerating bifurcate axon is associated with differential axonal transport of organelles. Dev Biol 124:35-40.

Goslin K, Banker G (1989) Experimental observations on the development of polarity by hippocampal neurons in culture. J Cell Biol 108:1507-1516.

Goslin K, Banker G (1991) Rat hippocampal neurons in low density culture. In: Culturing nerve cells (Banker G, Goslin K, eds), pp 252281. Cambridge, MA: MIT.

Hollenbeck PJ (1993) Products of endocytosis and autophagy are retrieved from axons by regulated retrograde organelle transport. J Cell Biol 121:305-315.

Jareb M, Banker G (1997) Inhibition of axonal growth by Brefeldin A in hippocampal neurons in culture. J Neurosci 17:8955-8963.

Lamoureux P, Buxbaum RE, Heidemann SE (1998) Axonal outgrowth of cultured neurons is not limited by growth cone competition. J Cell Sci 111:3245-3252.

Lin CH, Thompson CA, Forscher P (1994) Cytoskeletal reorganization underlying growth cone motility. Curr Opin Neurobiol 4:640-647.

Mandell JW, Banker GA (1996) A spatial gradient of tau phosphorylation in nascent axons. J Neurosci 16:5727-5740.

Mandell JW, Banker GA (1998) Selective blockade of axonogenesis in cultured hippocampal neurons by the tyrosine phosphatase inhibitor orthovanadate. J Neurobiol 35:17-28.

Marsh L, Letourneau PC (1984) Growth of neurites without filopodial or lamellipodial activity in the presence of cytochalasin B. J Cell Biol 99:2041-2047.

Mattson MP, Kater SB (1988) Isolated hippocampal neurons in cryopreserved long-term cultures: development of neuroarchitecture and sensitivity to NMDA. Int J Dev Neurosci 6:439-452.

Mattson MP, Partin J (1999) Evidence for mitochondrial control of neuronal polarity. J Neurosci Res 56:8-20.

Miller RH, Lasek RJ, Katz MJ (1987) Preferred microtubules for vesicle transport in lobster axons. Science 235:220-222.

Morris RL, Hollenbeck PJ (1993) The regulation of bidirectional mitochondrial transport is coordinated with axonal outgrowth. J Cell Sci 104:917-927.

Morris RL, Hollenbeck PJ (1995) Axonal transport of mitochondria along microtubules and f-actin in living vertebrate neurons. J Cell Biol 131:1315-1326.

Murphey RK, Lemere CA (1984) Competition controls the growth of an identified axonal arborization. Science 224:1352-1355.

O'Leary DDM, Bicknese AR, De Carlos JA, Heffner CD, Koester SE, Kutka LJ, Terashima T (1990) Target selection by cortical axons: alternative mechanisms to establish axonal connections in the developing brain. Cold Spring Harb Symp Quant Biol 55:453-468.

Ruthel G, Banker G (1999) Role of moving growth cone-like "wave" structures in the outgrowth of cultured hippocampal axons and dendrites. J Neurobiol 39:97-106.

Ruthel G, Hollenbeck PJ (1998) Mitochondrial sorting at branch points is coordinated with differential growth of axonal branches in cultured rat hippocampal neurons. Mol Biol Cell 9:406a.

Schneider G (1973) Early lesions of superior colliculus: factors affecting the formation of abnormal retinal projections. Brain Behav Evol 8:73-109.

Smalheiser NR, Crain SM (1984) The possible role of "sibling neurite bias" in the coordination of neurite extension, branching, and survival. J Neurobiol 15:517-529.

Stoeckli ET (1997) Molecular mechanisms of growth cone guidance: stop and go? Cell Tissue Res 290:441-449.

Tosney KW, Landmesser LT (1985) Growth cone morphology and trajectory in the lumbosacral region of the chick embryo. J Neurosci 5:2345-2358.

Yu W, Baas PW (1994) Changes in microtubule number and length during axon differentiation. J Neurosci 14:2818-2829.

Zheng JQ, Zheng Z, Poo M-M (1994) Long-range signaling in growing neurons after local elevation of cyclic AMP-dependent activity. J Cell Biol 6:1693-1701. 